OPEN ACCESS

Edited by:

Shicheng Chen,

Michigan State University,

United States

Reviewed by:

Gregory D. Wiens,

ARS-USDA, United States

Mark L. Lawrence,

Mississippi State University,

United States

*Correspondence:

Eric Duchaud

eric.duchaud@inra.fr

Specialty section:

This article was submitted to Microbial Physiology and Metabolism,

a section of the journal

Frontiers in Microbiology

Received: 22 June 2017

Accepted: 28 August 2017

Published: 12 September 2017

Citation:

Rochat T, Fujiwara-Nagata E, Calvez S, Dalsgaard I, Madsen L, Calteau A, Lunazzi A, Nicolas $P$,

Wiklund T, Bernardet J-F and

Duchaud E (2017) Genomic Characterization of Flavobacterium psychrophilum Serotypes and Development of a Multiplex PCR-Based Serotyping Scheme.

Front. Microbiol. 8:1752.

doi: 10.3389/fmicb.2017.01752

\section{Genomic Characterization of Flavobacterium psychrophilum Serotypes and Development of a Multiplex PCR-Based Serotyping Scheme}

\author{
Tatiana Rochat ${ }^{1}$, Erina Fujiwara-Nagata ${ }^{2}$, Ségolène Calvez ${ }^{3}$, Inger Dalsgaard ${ }^{4}$, \\ Lone Madsen ${ }^{4}$, Alexandra Calteau ${ }^{5}$, Aurélie Lunazzi ${ }^{1}$, Pierre Nicolas ${ }^{6}$, Tom Wiklund $^{7}$, \\ Jean-François Bernardet ${ }^{1}$ and Eric Duchaud ${ }^{1 *}$
}

' Virologie et Immunologie Moléculaires, Institut National de la Recherche Agronomique, Université Paris-Saclay, Jouy-en-Josas, France, ${ }^{2}$ Department of Fisheries, Kindai University, Nara, Japan, ${ }^{3}$ Biologie, Epidémiologie et Analyse du Risque, Institut National de la Recherche Agronomique, Oniris, Nantes, France, ${ }^{4}$ National Veterinary Institute, Technical University of Denmark, Kongens Lyngby, Denmark, ${ }^{5}$ CEA/Genoscope/LABGeM, CNRS-UMR8030, Université d'Evry Université Paris-Saclay, Evry, France, ${ }^{6}$ Mathématiques et Informatique Appliquées du Génome à l'Environnement, Institut National de la Recherche Agronomique, Université Paris-Saclay, Jouy-en-Josas, France, ${ }^{7}$ Laboratory of Aquatic

Pathobiology, Environmental and Marine Biology, Faculty of Science and Engineering, Åbo Akademi University, Turku, Finland

Flavobacterium psychrophilum is a devastating bacterial pathogen of salmonids reared in freshwater worldwide. So far, serological diversity between isolates has been described but the underlying molecular factors remain unknown. By combining complete genome sequence analysis and the serotyping method proposed by Lorenzen and Olesen (1997) for a set of 34 strains, we identified key molecular determinants of the serotypes. This knowledge allowed us to develop a robust multiplex PCR-based serotyping scheme, which was applied to 244 bacterial isolates. The results revealed a striking association between PCR-serotype and fish host species and illustrate the use of this approach as a simple and cost-effective method for the determination of F. psychrophilum serogroups. PCR-based serotyping could be a useful tool in a range of applications such as disease surveillance, selection of salmonids for bacterial coldwater disease resistance and future vaccine formulation.

Keywords: Flavobacterium psychrophilum, salmonid aquaculture, fish-pathogenic bacteria, genomics, serotype, MPCR

\section{INTRODUCTION}

Salmonids represent one of the most important fish groups in the aquaculture industry (FAO, 2016). However, the success and sustainability of salmonid aquaculture largely depend on disease control.

Flavobacterium psychrophilum, a bacterium belonging to the family Flavobacteriaceae, is considered one of the most important bacterial pathogens in freshwater salmonid aquaculture worldwide (Nematollahi et al., 2003). The two main clinical forms are rainbow trout fry syndrome (RTFS) and bacterial cold-water disease (BCWD) (Cipriano and Holt, 2005) that can both result in considerable economic losses (Antaya, 2008; Starliper, 2011). All salmonid fish, especially 
coho salmon (Oncorhynchus kisutch) and rainbow trout (Oncorhynchus mykiss) are susceptible to RTFS and BCWD, as well as ayu (Plecoglossus altivelis), a fish related to salmonids. These infections are associated with skin ulcers, necrotic myositis, septicemia as well as exophthalmia (Nematollahi et al., 2003). F. psychrophilum has occasionally been detected in nonsalmonid fish, samples of water, sediments and biofilms from rivers receiving outlet water from infected fish farms (Madetoja et al., 2002; Starliper, 2011). Despite extensive research on vaccine development (Plant et al., 2009; Gómez et al., 2014) and the recent marketing authorization of a vaccine in Chile, treatment still essentially relies on antibiotic administration (Schmidt et al., 2000). Diversity among F. psychrophilum isolates was reported at different levels, and a variety of typing schemes (e.g., biotypes, serotypes, and genotypes) was proposed accordingly. However, the correlation between these different schemes (Madsen and Dalsgaard, 2000; Madetoja et al., 2001; Ngo et al., 2017) and the impact of the different types on the onset and severity of the infection remain poorly understood.

Gram-negative bacteria have a complex set of surface polysaccharides that include the lipopolysaccharide (LPS) as well as capsular polysaccharides (CPS) or exopolysaccharides (EPS) (Raetz and Whitfield, 2002). These structures are thought to play important roles in the pathogenicity, immune escape, serum resistance, inflammation, adhesion and biofilm formation (Finlay and McFadden, 2006). Serotyping is based on the immunogenicity of various surface-exposed bacterial structures (e.g., LPS, capsular polysaccharides, flagella, peptidoglycan, etc.) biosynthesized by genes belonging to the accessory genome. This represents an advantage over many molecular typing methods (e.g., 16S rRNA sequencing, ribotyping, MLST) that rely on core genome genes, which are not phenotypically relevant determinants. LPS is one of the most pro-inflammatory compounds of Gram-negative bacteria typically consisting of three regions: a hydrophobic domain known as lipid A, a non-repeating "core" oligosaccharide, and a distal polysaccharide (or $O$-antigen). The $O$-antigen greatly varies between and within species, providing the main basis for many serotyping schemes. The structure of the $O$-polysaccharide of $F$. psychrophilum strain CSF259-93 has been solved and found to be an unbranched polymer of trisaccharide repeating units (MacLean et al., 2001). A $70 \mathrm{~kb}$ region (FP1299 to FP1234) encompassing genes predicted to be involved in the biosynthesis, export, modification and polymerization of polysaccharides was previously identified in the genome of $F$. psychrophilum JIP 02/86 (Duchaud et al., 2007). Several substitutions of genes enclosed in this region were noticed by comparing the genomes of strains CSF259-93 and JIP 02/86 (Wiens et al., 2014).

Serological differences among F. psychrophilum isolates were noticed since early studies and different serotyping protocols were proposed, resulting in the description of a varying number of serotypes. Wakabayashi et al. (1994) described two distinct serotypes (O-1 and O-2) among a collection of Japanese isolates; a third serotype (O-3) was added in 1999 (Izumi and Wakabayashi, 1999) and a fourth one (O-4) in 2003 (Izumi et al., 2003). In Denmark, Lorenzen and Olesen (1997) proposed a serotyping scheme based on three serogroups (Fp, Th and $\mathrm{Fp}^{\mathrm{T}}$ ). Using these schemes, some degree of association between serotypes and host fish species was observed. For example, strong relationship between host and $\mathrm{O}$ serotypes was reported, O-1, O-2, and O-3 corresponding to isolates infecting Coho salmon, ayu, and rainbow trout, respectively (Izumi and Wakabayashi, 1999). Moreover, the co-existence of genetically and serologically diverse isolates within individual farms was detected in Finland (Madetoja et al., 2001) and in the United Kingdom (Ngo et al., 2017). Finally, Mata et al. (2002) identified as much as seven different serovars (1-7) and also reported obvious links with host fish species. This serological diversity might have important consequences for the selection of appropriate candidate strain(s) for vaccine development (Gómez et al., 2014 and references therein), selective breeding of salmonids for increased disease resistance (Leeds et al., 2010), follow-up studies, epidemiological surveillance, disease control (Ngo et al., 2017), as well as for a better understanding of virulence and host resistance traits.

In the present study, we used the serotyping method proposed by Lorenzen and Olesen (1997) together with genome-wide association studies to identify key molecular determinants of serotypes. Accordingly, a multiplex PCR-based serotyping scheme (mPCR) was developed and successfully applied to a collection of 244 bacterial isolates.

\section{MATERIALS AND METHODS}

\section{Genome Comparisons}

The complete or draft genome sequences used in this study are listed in Supplementary Table S1. Genome comparisons were performed using the web interface MicroScope (Vallenet et al., 2017), which allows graphic visualization enhanced by a synchronized representation of synteny groups ${ }^{1}$. Comparison of the gene content between strains was done by pairwise proteome similarity search using BlastP Bidirectional Best Hit and the MicroScope default parameters (i.e., $>80 \%$ protein identity, $>80 \%$ coverage). Analysis of gene organization was conducted using syntons (i.e., maximal set of orthologous gene pairs displaying a conserved organization) constructed with MicroScope default parameters (i.e., orthologous gene set having the same local organization in two strains were computed using BlastP Bidirectional Best Hit or having at least 30\% identity on $80 \%$ of the shortest sequence and a genomic co-localization with allowed gap set to 5 genes). Evolutionary relationships between strains were analyzed using the DNA sequences of 1549 single-copy genes of the core genome identified by singlelinkage clustering ( $E$-value $\leq 1 e-5$ in blastp v2.2.26 pairwise proteome comparisons, alignment with amino-acid sequence identity $\geq 85 \%$ and length $\geq 70 \%$ of the shortest sequence). To mitigate the effect of recombination that introduces random and often large number of linked nucleotide differences per diversification event, a whole-genome MLST approach was

\footnotetext{
${ }^{1}$ http://www.genoscope.cns.fr/agc/mage/
} 
TABLE 1 | Oligonucleotides used in this study.

\begin{tabular}{llc}
\hline Name & Sequence & Amplicon size \\
\hline $\begin{array}{l}\text { ctrol_fw } \\
\text { ctrol_rev }\end{array}$ & AGCAAATTGGCTCTTTGG & $188 \mathrm{bp}$ \\
Type-1_fw & ACGTAACAACGCCACCAGTT & \\
Type-1_rev & GGGGAGTGGTTAGAACTGA & $549 \mathrm{bp}$ \\
Type-2_fw & TTGAACGAAACTTATATGGATAGA & $841 \mathrm{bp}$ \\
Type-2_rev & TTACCAAAGAGCCCTTTAGTG & \\
Type-3_fw & CGCCATGCAAGAAATTATT & $361 \mathrm{bp}$ \\
Type-3_rev & CCTGCGATCTCAACATATCA & \\
\hline
\end{tabular}

adopted (Jolley and Maiden, 2010). Pairwise distances were computed as the fraction of genes where alleles differ between two strains and a tree was then obtained and drawn using the 'nj' and 'plot.phylo' functions of R package 'ape' (Popescu et al., 2012).

\section{Bacterial Isolates and Serological Tests}

The F. psychrophilum isolates used in the present study are listed in Supplementary Table S1. The antisera were raised in rabbits against the serotypes $\mathrm{Fp}^{\mathrm{T}}\left(=\mathrm{NCIMB} 1947^{\mathrm{T}}\right), \mathrm{Fd}(=$ DIFR 950106-1/1), and Th (= DK002 = 900406-1/3) (Dalsgaard and Madsen, 2000). The slide agglutination tests were performed using cross-absorbed antisera as previously described (Lorenzen and Olesen, 1997). Briefly, bacteria were grown on tryptone yeast extract salts broth (Holt et al., 1993) with 1.1\% agar for 4 to 6 days. Bacterial colonies were suspended in sodium acetate buffer ( $\left.0.05 \mathrm{M} \mathrm{NaCO}_{2} \mathrm{CH}_{3}, 0.1 \mathrm{M} \mathrm{NaC} 1, \mathrm{pH} 7.5\right)$ and heated at $55^{\circ} \mathrm{C}$ for 10 to $15 \mathrm{~min}$, and $15 \mathrm{mM}$ sodium azide was subsequently added. Absorbed anti-Th, anti-Fd and anti-Fp ${ }^{T}$ antisera were used undiluted. Ten microliter of antiserum was allowed to react with 10 to $15 \mu \mathrm{L}$ of the bacterial suspensions on a slide using a gentle rocking motion. The reaction was recorded macroscopically against a dark background after 1 to $2 \mathrm{~min}$. Controls for autoagglutination were done in saline.

\section{Multiplex PCR}

DNA was extracted using a Wizard genomic DNA purification kit (Promega) according to the manufacturer's instructions. Primers (Table 1) were designed using Primer3 web version 4.0.0 2 according to the complete and draft genome sequences available and conservation of their sequences was verified. FP0711, a coregenome gene highly conserved in F. psychrophilum was used as a positive control for DNA amplification. MPCR reactions were performed using DreamTaq DNA polymerase, $10 \times$ DreamTaq buffer (20 mM $\mathrm{MgCl}_{2}$ ) (Thermo fisher scientific) and $10 \mu \mathrm{M}$ of each primer in a $50 \mu \mathrm{L}$ final reaction volume. The $\mathrm{mPCR}$ amplification mix was heated at $95^{\circ} \mathrm{C}$ for $5 \mathrm{~min}$, followed by 30 cycles of $95^{\circ} \mathrm{C}$ for $30 \mathrm{~s}, 52^{\circ} \mathrm{C}$ for $30 \mathrm{~s}, 72^{\circ} \mathrm{C}$ for $60 \mathrm{~s}$, and a final extension at $72^{\circ} \mathrm{C}$ for $10 \mathrm{~min}$. The amplified products were electrophoresed in $2.0 \%$ agarose gels run in $1 \times$ Tris-borate buffer with the GeneRuler DNA ladder mix (Thermo Fisher Scientific) as the molecular size standard.

\footnotetext{
${ }^{2}$ http://primer3.ut.ee
}

\section{Statistical Analysis}

The putative association between genotypes and host fish species was investigated using chi-squared tests. Post hoc tests were conducted by comparing chi-squared residual values with normal distribution (the corresponding $p$-values were adjusted for multiple testing using the Bonferroni correction procedure). These analyses were conducted with the R package.

\section{RESULTS}

\section{Comparative Genomics Identify Key Molecular Determinants of the F. psychrophilum Serotypes}

Using the slide agglutination test proposed by Lorenzen and Olesen (1997), serotyping was performed on the 34 strains which complete genomes were available in our laboratories. This serotyping scheme is able to discriminate 3 serotypes: $\mathrm{Fp}^{\mathrm{T}}$, Th, and $\mathrm{Fd}$, with some strains reacting with multiple antisera. The results are shown in Table 2. Strikingly, all strains but one belonging to CC-ST2/10 display the serotypes Fd or Th (Figure 1). However, the distribution of strains with these serotypes does not strictly follow the tentative phylogeny based on whole-genome MLST. For instance, strains FI056 and DK002, though tightly clustered in the phylogenetic tree, belong to serotypes $\mathrm{Fd}$ and $\mathrm{Th}$, respectively. Comparison of the gene content of these two strains revealed that they share 2243 genes (i.e., the core genome genes) but contain only four and two strain-specific genes, respectively. Two of them (FI056_50102 and DK002_320117) are co-localized, substituting each other, and lie in the predicted polysaccharide biosynthesis locus (Figure 2). FI056_50102 and DK002_320117 are orthologous genes of FP1290 and FPSM_02202, both encoding hypothetical proteins in strains JIP02/86 and CSF259-93, respectively. These genes are adjacent to $w b u A$ encoding a rhamnosyl transferase (Miyamoto et al., 2010), predicted to be involved in the $O$-antigen biosynthesis.

Extensive comparative genomic analysis of this locus revealed a globally well-conserved structural backbone. By grouping the strains harboring identical or nearly identical neighboring gene organization using FI056_50102/DK002_320117 as the seed, we detected another group of genomes containing a third gene, named FPC840_340035 in strain FPC840, that substitutes FI056_50102/DK002_320117 at the same chromosomal location (Figure 2). Based on the presence/absence of these three genes, four main genomic organizations, hereafter designated "Types," were identified. Strains FI056, DK002, and FPC840 are the typical examples of Type-1, Type-2, and Type- 3 , respectively. Type-0 encompasses a number of strains that do not belong to any of Types 1, 2 and 3 (i.e., not containing any genes related to FI056_50102, DK002_320117, or FPC840_340035) and displays a less-conserved genomic structure and a much broader genomic diversity. The type strain of F. psychrophilum, NCIMB $1947^{\mathrm{T}}$, belongs to this type.

Comparing the serotypes of the $34 \mathrm{~F}$. psychrophilum strains to their Type revealed a remarkable correlation (Table 2). Strikingly, 
TABLE 2 | Serotyping of F. psychrophilum isolates.

\begin{tabular}{|c|c|c|}
\hline Strain & Serotype & mPCR Type \\
\hline FI056 & $\mathrm{Fd}$ & 1 \\
\hline DIFR 950106-1/1* & $\mathrm{Fd}$ & 1 \\
\hline JIP 02/86 & $\mathrm{Fd}$ & 1 \\
\hline LM-01-Fp & $\mathrm{Fd}$ & 1 \\
\hline NO098 & $\mathrm{Fd}$ & 1 \\
\hline DK001 & $\mathrm{Fd}$ & 1 \\
\hline FI055 & $\mathrm{Fd}$ & 1 \\
\hline JIP 08/99 & $\mathrm{Fd}$ & 1 \\
\hline KU 061128-01 & $\mathrm{Fd}$ & 1 \\
\hline KU 051128-10 & $\mathrm{Fd}$ & 1 \\
\hline LVDJ XP189 & $\mathrm{Fd}-\mathrm{Fp}^{\top}$ & 1 \\
\hline $\mathrm{CH} 1895$ & Fd-Th-Fp' & 1 \\
\hline IT9 & auto-agglutination & 1 \\
\hline DK002** & Th & 2 \\
\hline Fl166 & Th & 2 \\
\hline $\mathrm{CH} 8$ & Th & 2 \\
\hline LM-02-Fp & Th & 2 \\
\hline FRGDSA 1882/11 & Th & 2 \\
\hline ITO2 & Th & 2 \\
\hline $\mathrm{NO042}$ & Th & 2 \\
\hline NO083 & Th & 2 \\
\hline NO014 & Th & 2 \\
\hline $\mathrm{FIO70}$ & Th-Fp' ${ }^{\top}$ & 2 \\
\hline $\mathrm{NCIMB} 1947^{\top * * *}$ & $F p^{\top}$ & 0 \\
\hline OSU THCO2-90 & $\mathrm{Fp}^{\top}$ & 0 \\
\hline DK150 & $\mathrm{Fp}^{\top}$ & 0 \\
\hline NOO4 & $\mathrm{Fp}^{\top}$ & 0 \\
\hline JIP16/00 & $\mathrm{Fp}^{\top}$ & 0 \\
\hline DK095 & $\mathrm{Fp}^{\top}$ & 0 \\
\hline FPC 831 & $\mathrm{Fp}^{\top}$ & 0 \\
\hline $\mathrm{Fl} 146$ & $\mathrm{Fp}^{\top}$ & 0 \\
\hline FPC 840 & $F p^{\top}$ & 3 \\
\hline KU 060626-04 & $\mathrm{Fp}^{\top}$ & 3 \\
\hline KU 060626-59 & $\mathrm{Fp}{ }^{\top}$ & 3 \\
\hline
\end{tabular}

* Strain DIFR 950106-1/1 was used to produce the anti-Fd antiserum.

** Strain DK002 = 900406-1/3 was used to produce the anti-Th antiserum.

*** Strain NCIMB $1947^{\top}$ was used to produce the anti-Fp ${ }^{T}$ antiserum.

all strains $(n=10)$ that only reacted with the anti-Fd antiserum belong to Type-1, all strains $(n=9)$ that only reacted with the anti-Th antiserum belong to Type- 2 , and all strains $(n=11)$ that only reacted with the anti-Fp ${ }^{\mathrm{T}}$ antiserum belong to Type- 0 (8 strains) or Type-3 (3 strains). A search for genes consistently present ( $>80 \%$ identity, $>80 \%$ coverage) in all strains belonging exclusively to serotype Fd (i.e., FI056, DIFR 950106-1/1, JIP 02/86, LM-01-Fp, NO098, DK001, FI055, JIP 08/99, KU 061128-1, and KU 051128-10) and absent from all strains belonging exclusively to serotype Th (i.e., DK002, FI166, CH8, LM-02-Fp, FRGDSA 1882/11, IT2, NO042, NO083, and NO014) revealed a unique gene (FI056_50102 and its orthologs from the above-mentioned genomes, i.e., IB65_06085, FP1290, LM01FP_150098, NO098_460117, DK001_50101, FI055_170072, JIP0899_1410015, KU06112801_120020, and KU05112810_320061, respectively). Conversely, a search for genes consistently present ( $>80 \%$ identity, $>80 \%$ coverage) in all strains belonging exclusively to serotype Th and absent in all strains belonging exclusively to serotype $\mathrm{Fd}$ revealed a unique gene (DK002_320117 and its orthologs from the above-mentioned genomes, i.e., FI166_350102, CH008_450117, LM02FP_380101, FRGDSA1882_70059, IT2_340117, NO042_ 670087, NO083_60118, and NO014_350089, respectively).

The following particular cases were noticed: strain LVDJ XP189 reacted with the anti-Fd and anti-Fp ${ }^{\mathrm{T}}$ antisera; strain $\mathrm{CH} 1895$ reacted with the anti-Fd, anti-Th and anti-Fp ${ }^{\mathrm{T}}$ antisera and displayed technical variability between replicates; and strain IT9 was untypable due to auto-aggregation; these three strains belong to Type-1. Finally, strain FI070 reacted with the anti-Th and anti-Fp ${ }^{\mathrm{T}}$ antisera and belongs to Type- 2 .

\section{Development of a Multiplex PCR}

Genes FI056_50102, DK002_320117 and FPC840_340035 do not display any primary sequence similarities. We therefore designed primers able to be used in a MPCR assay (Table 1). We included a positive control for PCR amplification that targets FP0711, a highly conserved gene ubiquitous in the F. psychrophilum genomes. To insure a straightforward discrimination of the types following gel electrophoresis, amplicons of 188, 361, 549 and 841 bp were selected for the positive control, Type-3, Type- 1 and Type-2, respectively. Because of the diversity of Type-0 loci and the lack of a specific gene, no specific primers were designed for the latter and the amplification of only the control testified for Type-0 (Figure 3).

\section{Screening of a F. psychrophilum Strain Collection Using Multiplex PCR}

The mPCR scheme was then used to screen a collection of 244 F. psychrophilum strains isolated from the fifties to 2015 from a wide variety of geographic areas and host fish species. All amplifications were successful and the results are show in Supplementary Table S2. This collection encompasses 28 strains that were included in the original paper of Lorenzen and Olesen (1997). A remarkable correlation between the previously reported serotypes obtained by these authors and the Types obtained using mPCR was observed for the 15 strains reacting with a unique antiserum using both agglutination and ELISA. Indeed: (i) the three strains (DIFR 911209-2, DIFR 930210-1, and DIFR 930413-1) that reacted exclusively with the anti-Fd antiserum belong to Type-1; (ii) the eleven strains (DIFR 900406-1, DIFR 910614-2, DIFR 910614-3, DIFR 910614-5, DIFR 911009-3, DIFR 911126-3, DIFR 930310-1, DIFR 930407-1, DIFR 930427-2, DIFR 930611-2, and Fi 147/93) that reacted exclusively with the anti-Th antiserum belong to Type-2; and (iii) the single strain (LFNW 16/90) that reacted exclusively with the anti-Fp ${ }^{T}$ antiserum belongs to Type- 0 . The situation of four cross-reacting isolates is more complex but in perfect agreement with Lorenzen and Olesen's observations: i.e., Fd-Fp ${ }^{\mathrm{T}}$ cross-reacting isolates using agglutination assay should be considered more closely related to $\mathrm{Fd}$ whereas $\mathrm{Th}-\mathrm{Fp}^{\mathrm{T}}$ cross-reacting isolates more closely related to Th. Using this interpretation, $\mathrm{mPCR}$ is consistent with the serotypes for four additional strains: (i) the three strains (LPAA 


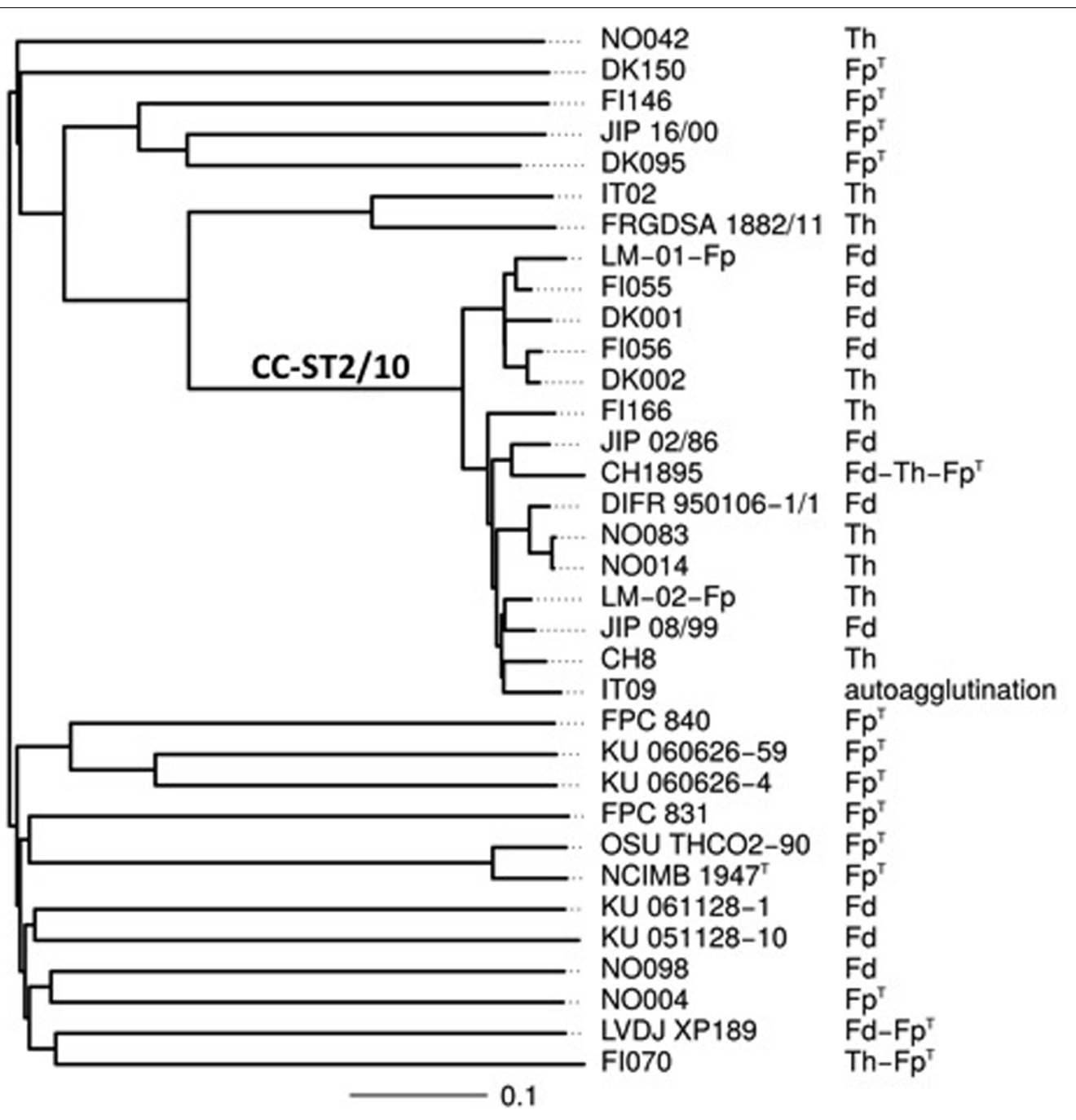

FIGURE 1 | Serotypes and evolutionary relationships between the 34 F. psychrophilum strains. Serotypes are shown on a tree attempting to capture the phylogenetic relationships between the 34 F. psychrophilum strains used in this study for which complete genomes were available. The tree was constructed based on whole-genome MLST profiles (see Materials and Methods for details). Distances are expressed in terms of the fraction of genes where alleles differ between strains. The branch that encompasses strains belonging to clonal complex 2/10 is indicated as CC-ST2/10.

11522, Fi 171/93, and Fi 206/93) that reacted with both antiFd and anti-Fp ${ }^{T}$ antisera in agglutination assay and exclusively with the anti-Fd antiserum in ELISA assay belong to Type-1 and (ii) the strain (DIFR 910516-1) that reacted with both antiTh and anti-Fp ${ }^{\mathrm{T}}$ antisera in agglutination assay and exclusively with the anti-Th antiserum in ELISA assay belongs to Type2. Finally, among the 28 isolates, the following particular cases (9 isolates) were noticed: (i) the two strains (LVDI 5/1 and Fi $196 / 93$ ) that reacted exclusively with the anti- $\mathrm{Fp}^{\mathrm{T}}$ antiserum in agglutination assay and that were untypable using ELISA belong to Type-1; (ii) the single strain (DIFR 910611-1) that reacted exclusively with the anti-Fp ${ }^{\mathrm{T}}$ antiserum in both agglutination and ELISA belongs to Type-2; (iii) among the three strains that reacted with both anti-Fd and anti-Th antisera in agglutination but only with anti-Th in ELISA, DIFR 930324-1 belongs to Type1 whereas Fi 88/93 and DIFR 910619-1 belong to Type-2; and finally (iv), three strains (USC PT 4.1, JIP 28/86 and LPAA 11524) that reacted with the anti-Fd, anti-Th and anti-Fp ${ }^{\mathrm{T}}$ antisera in agglutination assay but only with the anti-Th antiserum in ELISA belong to Type-1. In their publication, Lorenzen and Olesen (1997) concluded than the ELISA assay discriminate different serotypes most distinctly than agglutination assay. If we compare ELISA results reported by these authors with $\mathrm{mPCR}$ results, $91 \%$ of correlation was found (22 out of the 26 ELISA-typable strains).

Our data clearly show a significant association between the Types and the host fish species (Figure 4): 22 out of the 23 strains retrieved from Coho salmon belong to Type-0 ( $p$-value $=0), 59$ and 60 out of the 151 strains retrieved from rainbow trout belong to Type- 1 and to Type-2, respectively ( $p$-values $=1.8 e-7$ and $5.8 e-7)$ and all 35 strains retrieved from ayu belong to Type- 3 $(p$-value $=0)$. 
Type 1

Strains F1056, DK001, F1055, JIP 08/99, IT09, DIFR 950106-1/1

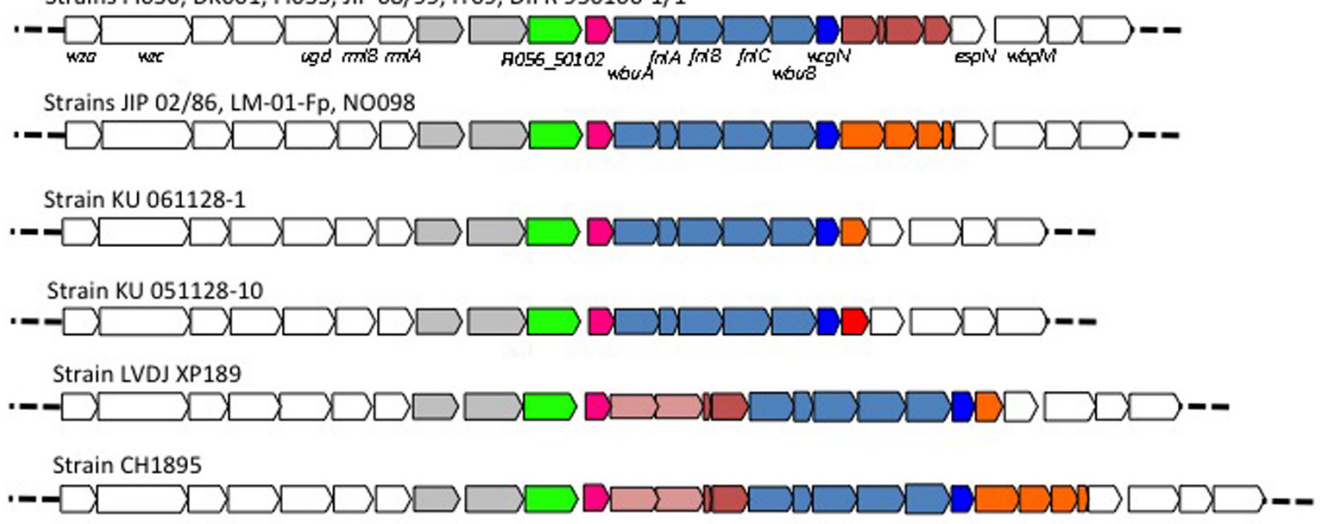

Type 2

Strains DK002, Fl166, CH8, LM-02-Fp, FRGDSA 1882/11, IT02, NO014, NO042, NO083

Strain Fl070

Type 3

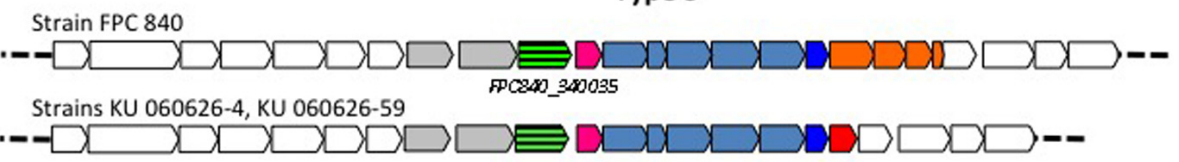

Type 0

Strain DK150

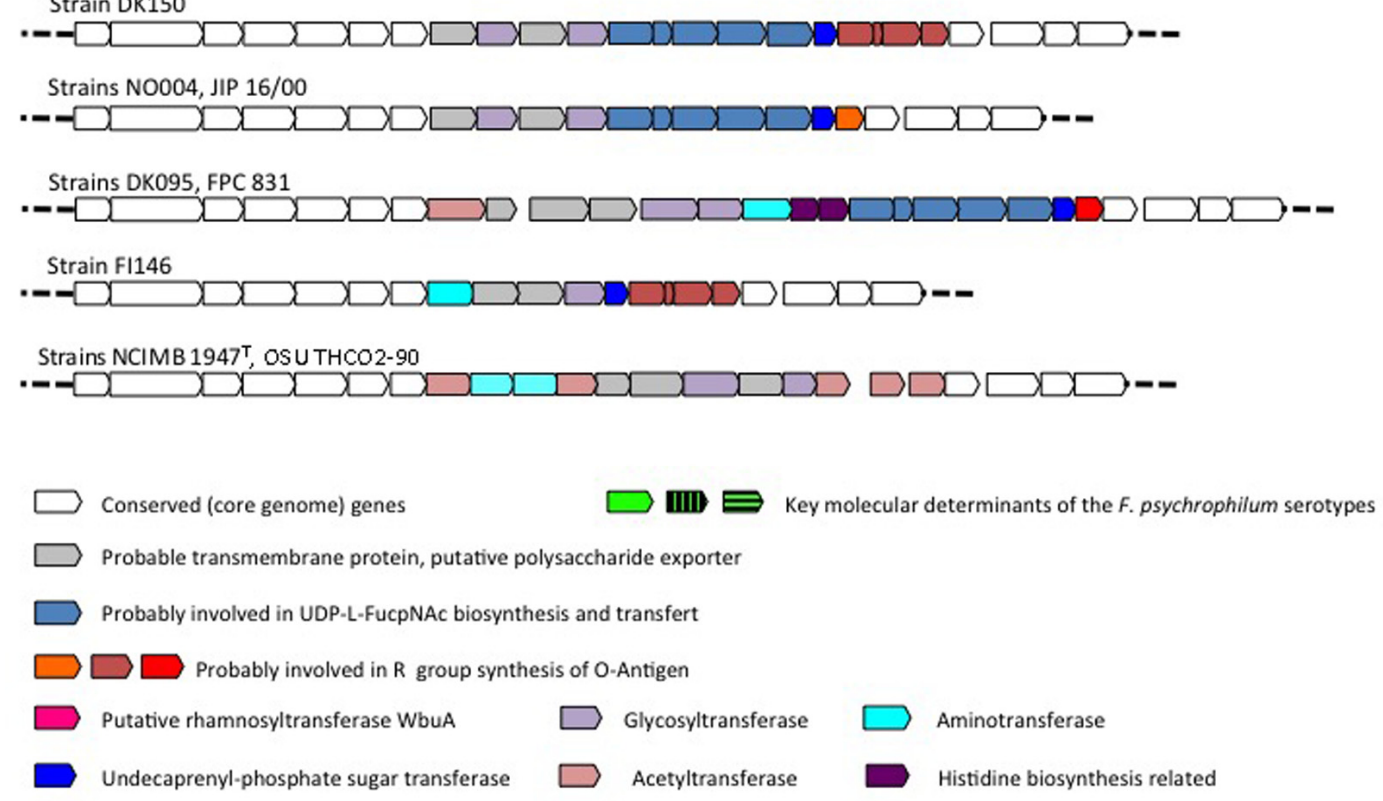

FIGURE 2 | Genomic organization of the polysaccharide encoding loci. The polysaccharide encoding loci were analyzed using the Microscope platform. Gene conservation was predicted using both homology and synteny criteria. White arrows represent core genome genes (i.e., genes conserved in the whole genome dataset which deduced proteins display $>80 \%$ identity over $>80 \%$ coverage) and colored arrows represent genes belonging to the variable genome (i.e., genes not conserved in the whole genome dataset). 


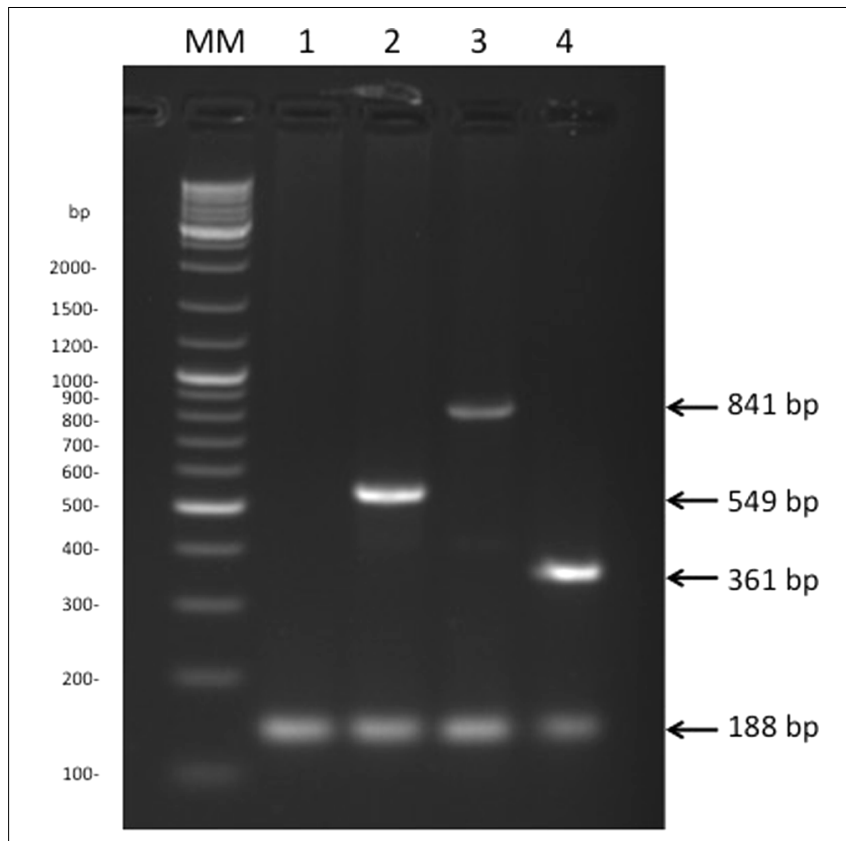

FIGURE 3 | Multiplex PCR. Migration of the PCR products after Multiplex PCR amplification: (MM) GeneRuler ${ }^{\text {TM }}$ molecular marker; (1) strain NCIMB $1947^{\top}$, revealing Type-0; (2) strain FI056 revealing Type-1; (3) strain DK002, revealing Type-2; (4) strain FPC 840, revealing Type-3.

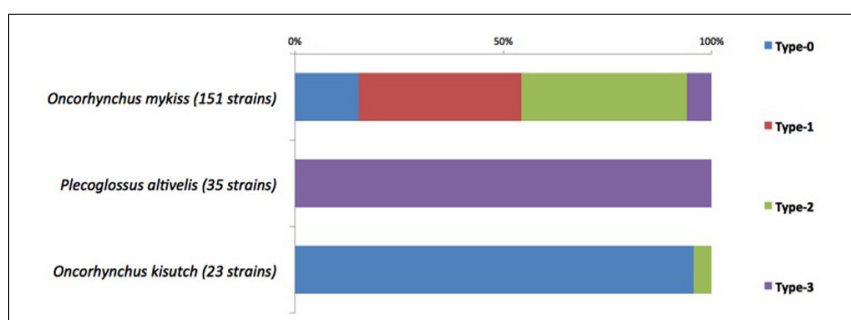

FIGURE 4 | Association between Types and host fish species.

\section{DISCUSSION}

Serological differences among F. psychrophilum isolates were reported since the pioneering studies in the nineties and different serotyping schemes were proposed (Wakabayashi et al., 1994; Lorenzen and Olesen, 1997; Mata et al., 2002). However, the conventional protocols for the serotyping of F. psychrophilum isolates available so far suffer several drawbacks such as the choice of the appropriate scheme, the need to raise antisera from animals, and the bias of human interpretation of agglutination results. In addition, some strains display auto-agglutination, lack of reactivity, or cross-reaction with multiple antisera that make laborious reciprocal absorptions against heterologous strains necessary. Moreover, inconsistencies between the results of agglutination and ELISA have been reported (Lorenzen and Olesen, 1997). Hence, serotyping by conventional serological methods is costly, labor-intensive and requires significant technical expertise. Therefore, molecular serotyping using mPCR is a relevant alternative to traditional serotyping, though identification of molecular markers that determine the serotypes is a prerequisite in order to target them with DNA probes. Indeed, mPCR serotyping assays have been developed for a number of bacterial pathogens such as Haemophilus parasuis, Pasteurella multocida, Salmonella sp., Streptococcus suis, S. pneumoniae and S. agalactiae (Kong et al., 2005; Liu et al., 2013; Harper et al., 2015; Ma et al., 2016).

We took advantage of the availability of the whole-genome sequences of $34 \mathrm{~F}$. psychrophilum isolates (Duchaud et al., in review) and of the previously published serotyping scheme of Lorenzen and Olesen (1997) to identify key molecular determinants of the serotypes. Focusing on strains FI056 and DK002 that are phylogenetically closely related but belong to two different serotypes (Fd and Th) (Figure 1), we identified the strain-specific genes FI056_50102 and DK002_320117 encompassed in the polysaccharide biosynthesis-encoding locus. Indeed, among the genes surrounding FI056_50102 or DK002_320117, some are predicted to be involved in the biosynthesis of the nucleotide-sugar precursors of $\mathrm{O}$-antigen ( $r m l A$ and $r m l B$ for the synthesis of dTDP-L-rhamnose; $w b p M$ for the synthesis of UDP-N-Acetyl-D-quinovosamine and $f \ln A$, $f \ln B$ and $f \ln C$ for the synthesis of UDP- $N$-Acetyl-L-fucosamine) whereas others encode the sugar transferases required for the assembly of lipid-linked O-repeat units ( $w b u A, w b u B$ and $w c g N$, the latter encoding the initial undecaprenyl-phosphate sugar transferase) (Figure 2). Previous studies aiming at identifying $F$. psychrophilum antigens recognized by polyclonal rabbit antisera have been performed (Crump et al., 2001, 2003). LPS-containing O-polysaccharide and core-region lipooligosaccharide have reported to be immunological relevant structures targeted by these sera. It is therefore tempting to speculate that the $O$-polysaccharide, predicted to greatly vary within species, provides the antigenic specificity of the serotyping scheme used in this study. So far, the $O$-polysaccharide structure has been solved for only one F. psychrophilum strain (CSF25993), identified as belonging to Type 2 by mPCR (MacLean et al., 2001). Extensive biochemical analyses will be required for each F. psychrophilum serogroup to formally identify the nature of the carbohydrates targeted by each anti-serum.

Genes FI056_50102/DK002_320117 were therefore promising candidate markers for serotype affiliation. Following a genomewide association study using the $34 \mathrm{~F}$. psychrophilum isolates, four Types were defined and Types 1 and 2 were firmly associated to Fp or Th serotypes (Table 2). Type 3 was not associated to an exclusive serotype in the scheme defined by Lorenzen and Olesen as $\mathrm{Fp}^{\mathrm{T}}$ serotype was shared between Type 0 and Type 3 isolates. However, its strong association with ayu (Figure 4 and Supplementary Table S2) indicates that Type 3 likely corresponds to the $\mathrm{O} 2$ serotype defined by Izumi and Wakabayashi (1999). Remarkably, neither the three genes located at this position (FI056_50102, DK002_320117 and FPC840_340035) nor their encoded proteins display any primary sequence identity. Initially annotated as "hypothetical protein," they are all predicted to be multipass transmembrane proteins, located in the inner membrane, with weak homologies to polysaccharide-biosynthesis proteins (e.g., O-antigen 
TABLE 3 | In silico prediction of protein function.

\begin{tabular}{|c|c|c|c|c|}
\hline Protein & $\begin{array}{l}\text { BlastP (\% identity; } \\
\text { Eval) }\end{array}$ & COGnitor & InterProScan & $\begin{array}{l}\text { HHpred on PfamA-30 } \\
\text { (amino-acid } \\
\text { positions; Eval) }\end{array}$ \\
\hline FI056_50102 & $\begin{array}{l}\text { A7M6Y2 } \\
\text { O-antigen polymerase } \\
\text { Wzy of Klebsiella } \\
\text { pneumoniae } \\
(22 \% ; 0.0000000006)\end{array}$ & $\begin{array}{l}\text { COG3307 } \\
\text { Lipid A core - } \\
\text { O-antigen ligase and } \\
\text { related enzymes }\end{array}$ & $\begin{array}{l}\text { PF13425 } \\
\text { O-antigen ligase-like } \\
\text { membrane protein }\end{array}$ & $\begin{array}{l}\text { PF13425 } \\
\text { O-antigen ligase-like } \\
\text { membrane protein } \\
\text { (5 to } 414 ; 8.7 \mathrm{E}-16 \text { ) }\end{array}$ \\
\hline DK002_320117 & $\begin{array}{l}\text { I6RSC6 } \\
\text { Polysaccharide } \\
\text { polymerase Wzy of } \\
\text { Streptococcus } \\
\text { pneumoniae } \\
\text { (20\%; 0.0000001) }\end{array}$ & No hit & No hit & $\begin{array}{l}\text { PF14296 } \\
\text { O-antigen } \\
\text { polysaccharide } \\
\text { polymerase Wzy } \\
\text { (4 to } 417 ; 1.8 \mathrm{E}-14 \text { ) }\end{array}$ \\
\hline FPC840_340035 & $\begin{array}{l}\text { I6RSC6 Polysaccharide } \\
\text { polymerase Wzy of } \\
\text { Streptococcus } \\
\text { pneumoniae } \\
\text { (23.27\%; 0.00002) }\end{array}$ & No hit & No hit & $\begin{array}{l}\text { PF01901 } \\
\text { Putative O-antigen } \\
\text { polymerase } \\
\text { (24 to 415; 9.1E-14) }\end{array}$ \\
\hline
\end{tabular}

polymerase, $\mathrm{O}$-antigen ligase) (Table 3 ). They likely perform the same general function (i.e., an unknown step in polysaccharide biosynthesis) but their activity should lead to structural variations such as the nature of the glycosidic bounds, additional sugar ramification(s), chain length, etc. As no obvious orthologous genes have been identified in strains belonging to Type 0 , one might hypothesize that other multipass transmembrane proteins encompassed in polysaccharide biosynthesis-encoding locus could be functional homologs in these strains. Because of the phylogenetic incongruence of these genes, one must conclude that gene exchange by recombination took place at this locus. Indeed, F. psychrophilum was found to have a very high homologous recombination rate that contributes to population diversification (Nicolas et al., 2008; Vos and Didelot, 2009).

Taking advantage of this sequence divergence, we developed a mPCR assay able to discriminate strains belonging to Types 1, 2, 3 and 0 . Our mPCR assay proved to be successful as all 244 strains tested were typable using this scheme (Supplementary Table S2). Among these strains, 28 were analyzed in the original paper of Lorenzen and Olesen (1997) and an outstanding correlation between the previously reported ELISA serotypes and the MPCR results was observed. The situation is more complex using agglutination assay. As a matter of fact, one should keep in mind that the antisera consist of polyclonal antibodies that were processed by multiple rounds of reciprocal absorptions against different heterologous strains. Therefore, cross-reactivity observed using slide-agglutination could be considered as a technical drawback, often noticed using such assays. Other variations in the locus (e.g., gene substitution, polymorphism or differences observed in other genes of the locus for some strains) may also impact the serotype and could explain the puzzling results that were obtained with the remaining crossreacting isolates, especially since as much as seven different serotypes were reported for F. psychrophilum using a broader scheme (Mata et al., 2002). Using the latter scheme, strain FPC840 belongs to serovar 7 and using Izumi's scheme it belongs to serovar O2. It is therefore tempting to speculate that Type3 described in this paper actually corresponds to serovar 7 according to Mata and to serovar $\mathrm{O} 2$ according to Izumi. Another example is strain LVDI 5/1 that corresponds to serovar 5 using Mata's scheme and to $\mathrm{Fp}^{\mathrm{T}}$ using Lorenzen and Olesen's (1997) scheme. An attempt to correlate all the previously published serotyping schemes with mPCR is proposed in Supplementary Table S3. Because $\mathrm{Fp}^{\mathrm{T}}$ encompasses many strains displaying a poorer conservation of the locus together with a wider genomic diversity, one might suspect that they could also belong to some other serovars observed by Mata et al. (2002) or to other, yet undescribed serovars. One should conclude that the current serotyping schemes may not cover all existing serotypes of F. psychrophilum and that WGS is likely a promising and efficient approach to address this issue. Accordingly, our mPCR assay could be enriched by new targets allowing the detection of additional serogroups in the future.

Our results also revealed a striking association between mPCR-serotype and fish host species. All strains retrieved from coho salmon but one belong to Type-0. The only exception is the Chilean isolate MHC $1710 \mathrm{~K}$ that belongs to Type 2 . As previously proposed, Chilean fish farming practices have contributed to create overlapping host ranges as most fish farms often house mixed stocks of different salmonid species including coho salmon and rainbow trout (Avendaño-Herrera et al., 2009, 2014). The vast majority of strains retrieved from rainbow trout (119 among 151) belong to Types- 1 and 2 . However, 23 strains belong to Type-0 (20 from France, 2 from Switzerland, and one from Denmark) and 9 strains to Type-3 (8 from France and one from Israel). It is noteworthy that strains of serotypes Th and Fd were reported to be predominant in rainbow trout and associated to disease outbreaks (Lorenzen and Olesen, 1997; Dalsgaard and Madsen, 2000) and to tend to be more virulent than $\mathrm{Fp}^{\mathrm{T}}$ isolates in experimental infection model using rainbow trout (Madsen and Dalsgaard, 2000; Madetoja et al., 2002). Considering that Types- 1 and -2 correspond to Fd and Th serotypes, our results 
showed that Th and Fd are equally predominant (40\%) in our rainbow trout set of isolates. Previous studies reported quite similar results, with serotype Th observed more frequently (60\%) in the United Kingdom (Ngo et al., 2017) and Fd occurring more frequently in Finland (Madetoja et al., 2002). Altogether, the mPCR typing method represents a promising epidemiological tool as it allows determining if an isolate belongs to one of the two serotypes associated to disease outbreaks in European fish farms. Finally, all strains retrieved from ayu or their eggs belong to Type-3. It is therefore very tempting to speculate that the nature of the Type provides a selective advantage according to the host that is infected. Nevertheless, these associations might also reflect the underlying genetic structure of the population without necessarily involving a selective advantage linked to the serotype. Indeed, MLST analysis of F. psychrophilum population structure has also identified clear associations between particular clonal complexes and some fish host (Nicolas et al., 2008). Our identification of the genetic determinants of the serotypes provides a basis for future studies aiming at clarifying the links between serotypes, population structure, and virulence.

As previously observed for other bacterial pathogens, mPCRbased serotyping methods often perform better than traditional serotyping in terms of detection rate, accuracy, reproducibility and compatibility of the results between different laboratories (Harper et al., 2015; Ma et al., 2016). Therefore, our mPCR assay may be readily used in large-scale studies aiming at fish farming improvement. Indeed, this straightforward mPCR-based serotyping procedure might be very useful for a variety of nonacademic purposes such as bacterial strain selection for vaccine development, selective breeding or epidemiological surveillance in fish farms.

\section{AUTHOR CONTRIBUTIONS}

TR: Substantial intellectual contribution throughout the study, data interpretation and manuscript preparation. EF-N, SC, and AL: Bacterial isolates management, DNA extraction and mPCR. ID and LM: Bacterial isolates management and serological

\section{REFERENCES}

Antaya, C. (2008). Current eco-economical impacts of Flavobacterium psychrophilum. MMG 445 Basic Biotechnol. eJ. 4, 16-21.

Aveñdano-Herrera, R., Houel, A., Irgang, R., Bernardet, J. F., Godoy, M., Nicolas, P., et al. (2014). Introduction, expansion and coexistence of epidemic Flavobacterium psychrophilum lineages in Chilean fish farms. Vet. Microbiol. 170, 298-306. doi: 10.1016/j.vetmic.2014.02.009

Avendaño-Herrera, R., Ilardi, P., and Fernández, J. (2009). "Significance of Flavobacterium diseases on salmonid farming in Chile," in Proceedings of the 2nd Conference on Members of the Genus Flavobacterium, Paris.

Cipriano, R. C., and Holt, R. A. (2005). Flavobacterium psychrophilum, cause of bacterial cold-water disease and rainbow trout fry syndrome. Fish Dis. Lealf. 86, $1-44$.

Crump, E. M., Perry, M. B., Clouthier, S. C., and Kay, W. W. (2001). Antigenic characterization of the fish pathogen Flavobacterium psychrophilum. Appl. Environ. Microbiol. 67, 750-759. doi: 10.1128/AEM.67.2.750-759.2001

Crump, E. M., Perry, M. B., Gale, S., Crawford, E., and Kay, W. W. (2003). Lipopolysaccharide $\mathrm{O}$-antigen antibody-based detection of the fish pathogen tests. AC and PN: Participation in genomic data analysis and handling. TW and J-FB: Participation in data interpretation. ED: Substantial intellectual contribution throughout the study, gene mining, interpretation of data, manuscript preparation and responsible for acquisition of funding. All authors read and approved the final manuscript.

\section{FUNDING}

The study was funded by the EU EMIDA ERA-NET project "Control Flavobacteriaceae Infections in European Fish farms". The France Génomique National infrastructure is funded as part of Investissement d'avenir program managed by Agence Nationale pour la Recherche (contract ANR-10-INBS-09).

\section{ACKNOWLEDGMENTS}

The authors are very grateful to Lisbeth Schade Hansen, Kári Karbech Mouritsen and Catherine Fournel for very skillful technical assistance; to Ellen Lorenzen, Ruben AvendañoHerrera, Mitsuru Eguchi for providing bacterial strains and to Luc Jouneau and Sébastien Bridel for help with statistical analysis. The LABGeM (CEA/IG/Genoscope \& CNRS UMR8030) and the France Génomique National infrastructure are acknowledged for support within the MicroScope annotation platform.

\section{SUPPLEMENTARY MATERIAL}

The Supplementary Material for this article can be found online at: http://journal.frontiersin.org/article/10.3389/fmicb. 2017.01752/full\#supplementary-material

TABLE S1 | Genome sequences used in this study.

TABLE S2 | Screening of the Flavobacterium psychrophilum strain collection using $\mathrm{MPCR}$.

TABLE S3 | Comparison of previously published serotyping schemes with mPCR.

Flavobacterium psychrophilum. J. Mol. Microbiol. Biotechnol. 6, 182-190. doi: 10.1159/000077249

Dalsgaard, I., and Madsen, L. (2000). Bacterial pathogens in rainbow trout, Oncorhynchus mykiss (Walbaum), reared at Danish freshwater farms. J. Fish Dis. 23, 199-209. doi: 10.1046/j.1365-2761.2000.00242.x

Duchaud, E., Boussaha, M., Loux, V., Bernardet, J. F., Michel, C., Kerouault, B., et al. (2007). Complete genome sequence of the fish pathogen Flavobacterium psychrophilum. Nat. Biotechnol. 25, 763-769. doi: 10.1038/ nbt1313

FAO (2016). The State of World Fisheries and Aquaculture. Available at: http: //www.fao.org/3/a-i5555e.pdf

Finlay, B. B., and McFadden, G. (2006). Anti-immunology: evasion of the host immune system by bacterial and viral pathogens. Cell 124, 767-782. doi: 10 . 1016/j.cell.2006.01.034

Gómez, E., Méndez, J., Cascales, D., and Guijarro, J. A. (2014). Flavobacterium psychrophilum vaccine development: a difficult task. Microb. Biotechnol. 7, 414-423. doi: 10.1111/1751-7915.12099

Harper, M., John, M., Turni, C., Edmunds, M., St Michael, F., Adler, B., et al. (2015). Development of a rapid multiplex PCR assay to genotype Pasteurella multocida 
strains by use of the lipopolysaccharide outer core biosynthesis locus. J. Clin. Microbiol. 53, 477-485. doi: 10.1128/JCM.02824-14

Holt, R. A., Rohovec, J. S., and Fryer, J. L. (1993). "Bacterial cold-water disease," in Bacterial Diseases of Fish, 1st Edn, eds V. Inglis, R. J. Roberts, and N. R. Bromage (New York, NY: Wiley-Blackwell), 3-22.

Izumi, S., Liu, H., Aranishi, F., and Wakabayashi, H. (2003). A novel serotype of Flavobacterium psychrophilum detected using antiserum against an isolate from amago, Oncorhynchus masou rhodurus Jordan \& Gilbert, in Japan. J. Fish Dis. 26, 677-680. doi: 10.1046/j.1365-2761.2003.00502.x

Izumi, S., and Wakabayashi, H. (1999). Further study on serotyping of Flavobacterium psychrophilum. Fish Pathol. 34, 89-90. doi: 10.3147/jsfp.34.89

Jolley, K. A., and Maiden, M. C. J. (2010). BIGSdb: scalable analysis of bacterial genome variation at the population level. BMC Bioinformatics 11:595. doi: 10 . 1186/1471-2105-11-595

Kong, F., Ma, L., and Gilbert, G. L. (2005). Simultaneous detection and serotype identification of Streptococcus agalactiae using multiplex PCR and reverse line blot hybridization. J. Med. Microbiol. 54, 1133-1138. doi: 10.1099/jmm.0. 46244-0

Leeds, T. D., Silverstein, J. T., Weber, G. M., Vallejo, R. L., Palti, Y., Rexroad, C. E., et al. (2010). Response to selection for bacterial cold water disease resistance in rainbow trout. J. Anim. Sci. 88, 1936-1946. doi: 10.2527/jas.2009-2538

Liu, Z., Zheng, H., Gottschalk, M., Bai, X., Lan, R., Ji, S., et al. (2013). Development of multiplex PCR assays for the identification of the 33 serotypes of Streptococcus suis. PLOS ONE 8:e72070. doi: 10.1371/journal.pone.0072070

Lorenzen, E., and Olesen, N. J. (1997). Characterization of isolates of Flavobacterium psychrophilum associated with coldwater disease or rainbow trout fry syndrome II: serological studies. Dis. Aquat. Organ. 31, 209-220. doi: 10.3354/dao031209

Ma, L., Wang, L., Chu, Y., Li, X., Cui, Y., Chen, S., et al. (2016). Characterization of Chinese Haemophilus parasuis isolates by traditional serotyping and molecular serotyping methods. PLOS ONE 11:e0168903. doi: 10.1371/journal.pone. 0168903

MacLean, L. L., Vinogradov, E., Crump, E. M., Perry, M. B., and Kay, W. W. (2001). The structure of the lipopolysaccharide $\mathrm{O}$-antigen produced by Flavobacterium psychrophilum (259-93). Eur. J. Biochem. 268, 2710-2716. doi: 10.1046/j.14321327.2001.02163.x

Madetoja, J., Dalsgaard, I., and Wiklund, T. (2002). Occurrence of Flavobacterium psychrophilum in fish-farming environments. Dis. Aquat. Organ. 52, 109-118. doi: 10.3354/dao052109

Madetoja, J., Hänninen, M. L., Hirvelä-Koski, V., Dalsgaard, I., and Wiklund, T. (2001). Phenotypic and genotypic characterization of Flavobacterium psychrophilum from Finnish fish farms. J. Fish Dis. 24, 469-479. doi: 10.1046/j.1365-2761.2001.00326.x

Madsen, L., and Dalsgaard, I. (2000). Comparative studies of Danish Flavobacterium psychrophilum isolates: ribotypes, plasmid profiles, serotypes and virulence. J. Fish Dis. 23, 211-218. doi: 10.1046/j.1365-2761.2000.00240.x

Mata, M., Skarmeta, A., and Santos, Y. (2002). A proposed serotyping system for Flavobacterium psychrophilum. Lett. Appl. Microbiol. 35, 166-170. doi: 10.1046/ j.1472-765X.2002.01157.x

Miyamoto, Y., Mukai, T., Naka, T., Fujiwara, N., Maeda, Y., Kai, M., et al. (2010). Novel rhamnosyltransferase involved in biosynthesis of serovar 4specific glycopeptidolipid from Mycobacterium avium complex. J. Bacteriol. 192, 5700-5708. doi: 10.1128/JB.00554-10
Nematollahi, A., Decostere, A., Pasmans, F., and Haesebrouck, F. (2003). Flavobacterium psychrophilum infections in salmonid fish. J. Fish Dis. 26, 563-574. doi: 10.1046/j.1365-2761.2003.00488.x

Ngo, T. P., Bartie, K. L., Thompson, K. D., Verner-Jeffreys, D. W., Hoare, R., and Adams, A. (2017). Genetic and serological diversity of Flavobacterium psychrophilum isolates from salmonids in United Kingdom. Vet. Microbiol. 201, 216-224. doi: 10.1016/j.vetmic.2017.01.032

Nicolas, P., Mondot, S., Achaz, G., Bouchenot, C., Bernardet, J. F., and Duchaud, E. (2008). Population structure of the fish-pathogenic bacterium Flavobacterium psychrophilum. Appl. Environ. Microbiol. 74, 3702-3709. doi: 10.1128/AEM. 00244-08

Plant, K. P., LaPatra, S. E., and Cain, K. D. (2009). Vaccination of rainbow trout, Oncorhynchus mykiss (Walbaum), with recombinant and DNA vaccines produced to Flavobacterium psychrophilum heat shock proteins 60 and 70 . J. Fish Dis. 32, 521-534. doi: 10.1111/j.1365-2761.2009.01008.x

Popescu, A.-A., Huber, K. T., and Paradis, E. (2012). ape 3.0: new tools for distance-based phylogenetics and evolutionary analysis in R. Bioinformatics 28, 1536-1537. doi: 10.1093/bioinformatics/bts184

Raetz, C. R., and Whitfield, C. (2002). Lipopolysaccharide endotoxins. Annu. Rev. Biochem. 71, 635-700. doi: 10.1146/annurev.biochem.71.110601.135414

Schmidt, A. S., Bruun, M. S., Dalsgaard, I., Pedersen, K., and Larsen, J. L. (2000). Occurrence of antimicrobial resistance in fish-pathogenic and environmental bacteria associated with four danish rainbow trout farms. Appl. Environ. Microbiol. 66, 4908-4915. doi: 10.1128/AEM.66.11.4908-4915.2000

Starliper, C. E. (2011). Bacterial coldwater disease of fishes caused by Flavobacterium psychrophilum. J. Adv. Res. 2, 97-108. doi: 10.1016/j.jare.2010. 04.001

Vallenet, D., Calteau, A., Cruveiller, S., Gachet, M., Lajus, A., Josso, A., et al. (2017). MicroScope in 2017: an expanding and evolving integrated resource for community expertise of microbial genomes. Nucleic Acids Res. 45, D517-D528. doi: 10.1093/nar/gkw1101

Vos, M., and Didelot, X. (2009). A comparison of homologous recombination rates in bacteria and archaea. ISME J. 3, 199-208. doi: 10.1038/ismej.2008.93

Wakabayashi, H., Toyama, T., and Iida, T. (1994). A study on serotyping of Cytophaga psychrophila isolated from fishes in Japan. Fish Pathol. 29, 101-104. doi: $10.3147 /$ jsfp. 29.101

Wiens, G. D., LaPatra, S. E., Welch, T. J., Rexroad, C. III, Call, D. R., Cain, K. D., et al. (2014). Complete genome sequence of Flavobacterium psychrophilum strain CSF259-93, used to select rainbow trout for increased genetic resistance against bacterial cold water disease. Genome Announc. 2:e00889-14. doi: 10 . 1128/genomeA.00889-14

Conflict of Interest Statement: The authors declare that the research was conducted in the absence of any commercial or financial relationships that could be construed as a potential conflict of interest.

Copyright (C) 2017 Rochat, Fujiwara-Nagata, Calvez, Dalsgaard, Madsen, Calteau, Lunazzi, Nicolas, Wiklund, Bernardet and Duchaud. This is an open-access article distributed under the terms of the Creative Commons Attribution License (CC BY). The use, distribution or reproduction in other forums is permitted, provided the original author(s) or licensor are credited and that the original publication in this journal is cited, in accordance with accepted academic practice. No use, distribution or reproduction is permitted which does not comply with these terms. 